\title{
Complications in the 90-day postoperative period following kidney transplant and the relationship of the Charlson Comorbidity Index
}

Max A. Levine, $\mathrm{MD}^{1}$; Trevor Schuler, $\mathrm{MD}^{1}$; Sita Gourishankar, MD, $\mathrm{MSc}^{2}$
${ }^{1}$ Division of Urology; ${ }^{2}$ Department of Nephrology; University of Alberta, Edmonton, AB, Canada

Cite as: Can Urol Assoc J 2017; Epub ahead of print. http://dx.doi.org/10.5489/cuaj.4378

Published online November 1, 2017

$* * *$

\section{Abstract}

Introduction: Renal transplant experiences widespread success, but little is published regarding the postoperative complications. The Charlson Comorbidity Index (CCI) is a system of mortality risk assessment. Our purpose is to assess the 90-day postoperative complications after renal transplantation. The secondary objective is to clarify whether CCI predicts complications. We hypothesized increased CCI corresponds to worse complication on the Clavien scale.

Methods: This is a retrospective analysis of renal recipients at our institution (2011-2013), who were $\geq 18$ years old and received complete follow up. CCI, age, gender, body mass index (BMI), and graft type were extracted from the electronic medical records. Complications were scored using the Clavien scale. Descriptive statistics and logistic regression were used to analyze 198 patients.

Results: The mean age was 53 (standard deviation [SD] 14), mean BMI 27.4 (SD 14), median CCI 1. Grade 2 or higher (significant) complications occurred in $60 \%$ of patients and Grade $3 \mathrm{~b}$ or higher (severe) in $15 \%$ of patients in the 90 -day postoperative period. Sixty-eight different complications were identified, the most common being blood transfusion (19\%). Logistic regression suggests a predictive value of CCI (odds ratio [OR] 1.70; 95\% confidence interval [CI] 1.3-2.3) for severe complications, with DM and PVD conferring increased risk.

Conclusions: Renal transplant carries significant risk. This data can be used to improve patient counselling on the likely postoperative course. Study limitations include the retrospective design, predisposing to potential bias in data capture. 


\section{Introduction}

Renal transplantation is the gold standard therapy for managing end stage renal disease (ESRD). The long-term survival of transplanted kidneys has continued to improve over time attributable to improved immunosuppressant regimens and advancements in organ storage technology. ${ }^{1,2}$ Despite the success of renal transplantation, postoperative complications can be expected and are not categorically benign. Patients with ESRD often possess multiple co-morbidities including anemia, diabetes, peripheral vascular disease and cardiovascular disease. In addition, donated kidneys are a scarce resource and optimizing the allocation while minimizing the risk of graft loss is paramount.

The need to objectively assess the degree of patient co-morbidity has led to the development of several validated tools. The Charlson Co-morbidity Index (CCI) is a common and widely accepted instrument used to predict 10 -year mortality. ${ }^{3}$ The CCI has been further validated in the ESRD population to predict 10-year mortality for those on dialysis as well as in recipients of kidney transplant. ${ }^{4-6}$

Reports on kidney transplant outcomes largely focus on the rates of graft and/or patient survival. ${ }^{4,7,8}$ In contrast, patient morbidity in the post-operative period is poorly described in the literature. Reports on kidney transplant complications are sparse and generally reported without standardized definitions or severity. ${ }^{9,10}$ This deficiency in the literature may be due to a lack of uniform descriptions of what defines a complication. Further, the significance of different events in the post-operative period varies depending on the patient population and procedure undertaken, making quantification of morbidity difficult. Several authors have subsequently developed reports defining post-operative complications and their respective severities for specific surgical procedures, including pancreaticoduodenostomy and liver transplant. ${ }^{11,12}$

The objectives of this study are two fold. Firstly, to quantify the severity and nature of the 90-day post-operative complications of kidney transplant at our institution. Second, to assess the relationship between pre-operative co-morbidity, as defined by the CCI, and post-operative complications outlined by the Clavien-Dindo classification. We hypothesize that the severity of complications in transplant recipients will be commensurate with reports in the literature for other major surgical procedures and the CCI will strongly correlate with the rate and severity of post-operative complications.

\section{Methods}

A retrospective review of renal transplants at our institution from 2011-2013 was undertaken. Our centre services a population of approximately 2-3 million people, comprised of those living in northern and central Alberta, northwestern Saskatchewan, northeastern British Columbia and the Northwest Territories. We included all kidney transplant recipients 18 years or older who received follow-up transplant care in Edmonton, AB Canada. We excluded pediatric recipients, those receiving simultaneous kidney-pancreas transplants, and patients who were followed by nephrologists outside of our institution. The research protocol was approved by the Human Research Ethics Board at the University of Alberta. 
Data was abstracted from the Organ Transplant Tracking Record (OTTR ${ }^{\circledR}$ ) transplant electronic medical record (EMR), the in-house EMR used to document patient interactions with transplant health care providers. Patient information is linked to the health care profile on the provincial health record, creating a robust compilation of medical information. A query into the EMR for all recipients of a kidney from January 1, 2011 to December 31, 2013 provided a list of potential patients for review. Patient files meeting inclusion criteria were fully analyzed. Demographic information, including age at transplant, gender, body mass index (BMI), cause of ESRD, type of transplanted kidney and date of transplant were collected. A complete list of documented comorbidities was complied from the EMR by analyzing multiple visits with the transplant team to ensure completeness. The Charlson Co-morbidity Index (CCI) was calculated for each patient. ${ }^{13}$ Table 3 outlines the CCI categories.

Complications were identified through review of discharge summaries post-transplant, as well as any subsequent admission histories, emergency room visit records, and/or subsequent discharge summaries created for the patient in the 90 day post-operative period. Clinic progress notes, compiled by Nephrologists, Registered Nurses, Dieticians, and Social Workers, as part of routine follow-up care, were reviewed for identification of potential complications. This multidisciplinary lens on the 90 day post-operative period improved the robustness of our data collection despite our retrospective design.

Identified events potentially representing complications were documented and reviewed by the authors as a group to confirm the designation of complication. Of note, we assessed Delayed Graft Function (DGF; defined at our institution as a decrease in serum creatinine of $<10 \%$ in the first week or requiring hemodialysis within the first week of transplant) was not a complication unless dialysis was indicated for the treatment of life-threatening hyperkalemia or volume overload. This distinction was made because these patients are on dialysis pre-operatively, therefore DGF is not a new “complication” but rather a failure or delay in the realization of the outcome of the surgical intervention. Complications were then scored using the most recently published iteration of the Clavien-Dindo complication scale. ${ }^{14}$ The Clavien-Dindo scale is a validated tool that rates complications on a scale of 1-5 based on the intervention required to address them ${ }^{14}$. We defined a "severe" complication as Grade $3 b$ or higher, and a "significant" complication as Grade 2 or higher.

Statistical analysis was carried out on SPSS (C) version 22.0 software. Descriptive statistics of demographic information were calculated, and to identify risk factors associated with the development of a "severe" post-operative complication, univariate and multivariate step-wise logistic regression analysis were used. Significance was set at an $\alpha=0.05$.

\section{Results}

A total of 198 patients met inclusion criteria for analysis. The demographic information is shown in Table 1. Most recipients were male (136/198), and the population was mildly overweight (average BMI = 27.4). Table 2 displays the frequency of CCI scores. Most patients carried a mild degree of co-morbid illness, with the median CCI = $3(\mathrm{IQR}=1)$, and most had a CCI of 2 or 3 
(150/198). The distribution of patients populating each category of the CCI is shown in Table 3. Of note, diabetes mellitus was the most frequent CCI factor, other than moderate to severe chronic kidney disease, contributing to co-morbidity.

Figure 1 displays the number of patients experiencing each Clavien grade of complication. Overall, $60 \%$ of transplant recipients experienced at least one significant complication. Furthermore, 15\% of patients experienced a "severe" complication, with 1 death occurring in the 90-day post-operative period (cardiac arrest at home day 89 post-transplant; Male, age $=38$, CCI $=5$ (DM [2pts], ESRD [2pts], PVD [1pt], Living donor); no other grafts were lost in the 90-day post-operative period. Sixty eight different complications were identified and are presented in Table 4. Most complications occurred infrequently, however certain events appeared with greater regularity. These were blood transfusion (19\%), diarrhea from immunosuppressant medication (16\%), urinary tract infection (UTI) (6\%), de novo hypertension (4\%), ileus (4\%), symptomatic perigraft fluid collections (lymphocele, hematoma) (4\%). Among those patients who received a blood transfusion the mean (s.d.) pre-op hemoglobin was 105 (14.5) g/L while the mean (s.d.) pre-op hemoglobin in those spared a transfusion was 109 (15.1) g/L ( $\mathrm{p}=0.18)$.

Logistic regression analysis was used to assess the impact of pre-operative characteristics in determining the risk of development of a severe complication. Among age, gender, BMI, and CCI, only the CCI was associated with a significantly increased risk of severe complication (O.R. = 1.7; 95\% CI 1.3-2.3). The impact of BMI approached significance (O.R. $=1.09 ; 95 \% \mathrm{CI}$ 0.99-1.18), but the interaction of BMI and CCI was not significant (O.R. $=1.01$; 95\% CI 0.951.08). The cause of ESRD and type of kidney donated (living, donation after cardiac death, neurologic death donor) were not significantly associated with the occurrence of severe complications (data not shown).

To further elucidate the contributing factors for the development of severe complications, univariate logistic regression analysis between individual CCI categories and the rate of complications was performed, and subsequent multivariate logistic regression analysis was performed on each CCI category of significance. Univariate regression suggested the history of diabetes mellitus (DM), peripheral vascular disease (PVD), chronic obstructive pulmonary disease, and stroke may be associated with increased risk of severe complication. Subsequent multivariate logistic regression analysis revealed only DM (O.R. $=2.9 ; 95 \%$ CI 1.3-6.9) and PVD (O.R. $=4.4$; 95\% CI 1.0-18.9) were significantly predictive of severe complications.

\section{Discussion}

Kidney transplantation remains the optimal treatment for renal replacement therapy in those with ESRD in terms of both quality and quantity of life. ${ }^{15}$ While outcomes of kidney transplant with respect to graft and patient survival are generally excellent, the morbidity associated with the surgery itself is significant. In our study, more than half of patients who underwent a kidney transplant experienced a significant post-operative complication. Specifically, $60 \%$ of patients experienced a Clavien-Dindo Grade 2 or worse complication, with 15\% experiencing a severe complication. Of note, nearly 1 in 5 patients required blood transfusion after surgery. 
The morbidity associated with kidney transplant is on par with other morbid urologic procedures. Fairey et al (2008) found that in patients undergoing radical cystectomy with urinary diversion, $47 \%$ of patients experience an early post operative complication and $25 \%$ of patients experience a major early complication. ${ }^{16}$ In this retrospective analysis, level of co-morbidity, as categorized by the ACE-27 score, was associated with the risk of development of minor and major postoperative complications, as well as 90-day mortality. The use of different instruments to evaluate co-morbidity precludes direct comparison of absolute increases in risk of complication, but the qualitative trend is reproduced by our data: higher co-morbidity correlates with worse complications.

Other reports available on the rates of complication in kidney transplant appear to underestimate risk when compared to our data. Wolff et al (2014) retrospectively reported on the rates of complications after kidney transplant over 36 years at their institution and found that $24 \%$ of patients experienced at least one complication, whereas our data found a $60 \%$ rate of any significant complication. ${ }^{9}$ These authors defined a complication as the occurrence of one of six classes of events stemming from the graft itself: hemorrhage requiring reoperation, lymphocele requiring intervention, infection of any kind in the surgical site, complications of the ureter or ureteric anastamosis, vascular anastomosis complications, and neighboring organ injury. ${ }^{9}$ No differentiation was made between the severity of complications or quantification of other perioperative events, such as myocardial infarction or venous thromboembolism, and thus were not included in their analysis. Despite capturing a wider breadth of post-operative events as complications, our recorded rates of complication as defined by Wolff and colleagues are fewer. In our series the rate of comparable complications was only $15.2 \%$. This lower number likely reflects the contemporary nature of our data in contrast to their historical data on surgical outcomes. This observation highlights the potential value of standardized reportable complication systems.

Issues with standardized reporting of complications are evident in other reports on transplant outcomes. In a report of 90-day complications after simultaneous kidney-pancreas (KP) transplantation, 23 of 112 (21\%) of recipients experienced a complication of Clavien-Dindo Grade 2 or higher. ${ }^{10}$ These results appear to contrast with our results. Like Wolff et al (2014), these authors limit their reporting of post operative complications to those directly related to the graft: graft dysfunction, ureteral complications, infection of graft, perigraft fluid collections, and vascular thrombosis. Patients experience all complications, not just ones stemming from the graft itself; the omission of other relevant perioperative events significantly underestimates the risk of complications related to kidney transplant potentially experienced by recipients. The importance of recognizing more "minor" complications can aid in managing patient expectations.

In a report by Clavien et al (1994), the reported rate of any post-operative complication in liver transplant recipients was $99 \%{ }^{12}$ The authors report the rates of complications using a scoring system modified from the original Clavien-Dindo classification that is specific for commonly encountered complications after liver transplant. The use of a novel and procedure 
specific complication scale is justified as the standard post operative course for liver transplant involves routine intensive care unit admission and invasive cardiovascular monitoring that most other surgical procedures do not usually require.

In a similar vein, DeOliviera et al (2006) adapted the Clavien-Dindo scale to accommodate the commonly encountered complications in pancreatic surgery. ${ }^{11}$ Within the Clavien-Dindo grades, the severity of pancreatic surgery-specific complications were scaled according to the relative impact the complication had. For example, the authors report pancreatic fistula occurring in $9 \%$ (57/633) of patients, but the complication was scored as Grade 2 in 33 (5\%), Grade 3a in 15 (2\%), and Grade 5 in 2 patients (0.3\%). The weight ascribed to a given complication is dependent on the procedure performed. Our study attempted to slightly modify the definition of post-operative events while maintaining alignment with the Clavien-Dindo scale. Specifically, the occurrence of DGF and the associated dialysis that ensues was not in and of itself considered a complication unless it treated a life-threatening event (i.e. hyperkalemia requiring urgent dialysis - a Grade 4 complication according to Dindo et al [2004] ${ }^{14}$ ).

The present study does suffer from some limitations. The retrospective nature of the analysis creates the possibility of certain biases, however this is minimized by the use of multiple data sources. Co-morbidities used to calculate CCI were only included if they were documented in the EMR, potentially underreporting well managed or undisclosed co-morbid conditions. However, the integrative and multi-disciplinary nature of patient care pre- and post-transplant reduces this risk.

\section{Conclusion}

To our knowledge, this is the most in depth report of 90-day post-operative complications available for kidney transplant surgery. The procedure, despite its excellent long-term successes, carries significant morbidity, with a nearly 1 in 5 chance of requiring blood transfusion, and a 1 in 6 chance of Clavien-Dindo Grade 3b or worse complication. This information is important for patient counseling pre-operatively. It provides a realistic rate of complications, beyond those that threaten the graft, that should be disclosed and discussed with patients. As well, individual patient risk of severe complication may be anticipated by the presence of certain factors, namely higher CCI, and more specifically the presence of DM and PVD, which may help inform decisions on pre-emptive usage of ICU or intermediate care beds.

Complication reporting suffers in the literature due to non-standardization of case definitions and incomplete reporting of all relevant perioperative events. Other authors ${ }^{11,12}$ have demonstrated the utility of developing procedure-specific definitions of complications that more accurately report on the relative severity of a complication in context of the usual post-operative course. The development of similar procedure-specific definitions of complications and their relative severities is needed for kidney transplantation as well. With improved reporting on complications, transplant program quality assessment and outcomes research can be better appraised and improved upon in the future. 


\section{References}

1. Sedigh A, Tufveson G, Backman L, et al. Initial experience with hypothermic machine perfusion of kidneys from deceased donors in the uppsala region in sweden. Transplant Proc. 2013;45(3):1168-1171.

2. Bathini V, McGregor T, McAlister VC et al. Renal perfusion pump vs cold storage for donation after cardiac death kidneys: A systematic review. J Urol. 2013;189(6):22142220.

3. Charlson ME, Pompei P, Ales KL et al. A new method of classifying prognostic comorbidity in longitudinal studies: Development and validation. J Chronic Dis. 1987;40(5):373-383.

4. Grosso G, Corona D, Mistretta A, et al. Predictive value of the charlson comorbidity index in kidney transplantation. Transplant Proc. 2012;44(7):1859-1863.

5. Jassal SV, Schaubel DE, Fenton SS. Baseline comorbidity in kidney transplant recipients: A comparison of comorbidity indices. Am J Kidney Dis. 2005;46(1):136-142.

6. Hemmelgarn BR, Manns BJ, Quan $\mathrm{H}$ et al. Adapting the charlson comorbidity index for use in patients with ESRD. Am J Kidney Dis 2003;42(1):125-132.

7. Beddhu S, Bruns FJ, Saul M, et al. A simple comorbidity scale predicts clinical outcomes and costs in dialysis patients. Am J Med. 2000;108(8):609-613.

8. Mirzaee M, Azmandian J, Zeraati $\mathrm{H}$, et al. Patient survival in renal allograft failure: A time-dependent analysis. Nephrourol Mon. 2013;6(1):e13589.

9. Wolff T, Schumacher M, Dell-Kuster S, et al. Surgical complications in kidney transplantation: No evidence for a learning curve. J Surg Educ. 2014;71(5):748-55.

10. Grochowiecki T, Gałązka Z, Madej K, et al. Early complications related to the transplanted kidney after simultaneous pancreas and kidney transplantation. Transplant Proc. 2014;46(8):2815-2817.

11. DeOliveira ML, Winter JM, Schafer M, et al. Assessment of complications after pancreatic surgery: A novel grading system applied to 633 patients undergoing pancreaticoduodenectomy. Ann Surg. 2006;244(6):931-7; discussion 937-9.

12. Clavien PA, Camargo CA,Jr, Croxford R, et al. Definition and classification of negative outcomes in solid organ transplantation. application in liver transplantation. Ann Surg. 1994;220(2):109-120.

13. Fried L, Bernardini J, Piraino B. Charlson comorbidity index as a predictor of outcomes in incident peritoneal dialysis patients. Am J Kidney Dis. 2001;37(2):337-342.

14. Dindo D, Demartines N, Clavien PA. Classification of surgical complications: A new proposal with evaluation in a cohort of 6336 patients and results of a survey. Ann Surg. 2004;240(2):205-213.

15. Huang Y, Samaniego M. Preemptive kidney transplantation: Has it come of age? Nephrol Ther. 2012;8(6):428-432.

16. Fairey A, Chetner M, Metcalfe J, et al. Associations among age, comorbidity and clinical outcomes after radical cystectomy: Results from the alberta urology institute radical 
cystectomy database. J Urol. 2008;180(1):128-134. 


\section{Figures and Tables}

Fig. 1. Percentage of patients experiencing each Clavien-Dindo grade of complication.

Levine 2016 - 90-day Post-operative Complications of Renal Transplant

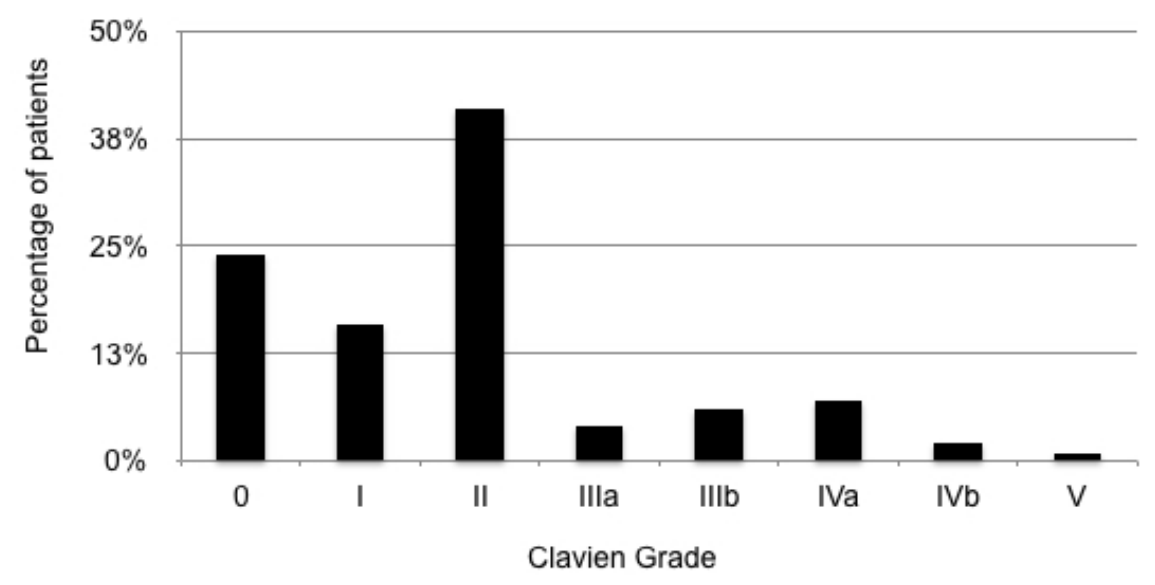

\begin{tabular}{|l|c|c|}
\hline \multicolumn{3}{|l|}{ Table 1. Demographic information } \\
\hline $\mathrm{N}$ & 198 & \\
\hline Age (mean, SD) & 52 & 14 \\
\hline No. male & 136 & \\
\hline No. female & 62 & \\
\hline BMI (mean, SD) & 27.4 & 4.9 \\
\hline Donor type & & \\
\hline $\begin{array}{l}\text { Live donor/paired } \\
\text { exchange }\end{array}$ & 77 & \\
\hline Cardiac death & 9 & \\
\hline Neurologic death & 112 & \\
\hline
\end{tabular}




\begin{tabular}{|c|c|c|c|}
\hline \multicolumn{5}{|c|}{ Table 2. Frequency of Charlson Comorbidity Index (CCI) scores } \\
\hline CCI score frequencies & & & \\
\hline Median (IQR) & 3 & 1 & \\
\hline CCI score & $\mathbf{n}$ & \% of patients & cumulative \% \\
\hline 0 & 0 & $0.0 \%$ & $0.0 \%$ \\
\hline 1 & 0 & $0.0 \%$ & $0.0 \%$ \\
\hline 2 & 90 & $45.5 \%$ & $45.5 \%$ \\
\hline 3 & 60 & $30.3 \%$ & $75.8 \%$ \\
\hline 4 & 30 & $15.2 \%$ & $90.9 \%$ \\
\hline 5 & 8 & $4.0 \%$ & $94.9 \%$ \\
\hline 6 & 7 & $3.5 \%$ & $98.5 \%$ \\
\hline 7 & 1 & $0.5 \%$ & $99.0 \%$ \\
\hline 8 & 2 & $1.0 \%$ & $100.0 \%$ \\
\hline
\end{tabular}

IQR: interquartile range. 


\begin{tabular}{|l|c|}
\hline \multicolumn{2}{|l|}{$\begin{array}{l}\text { Table 3. Breakdown of Charlson Comorbidity Index categories and frequency of } \\
\text { patients populating each category }\end{array}$} \\
\hline CCI categories & N \\
\hline 1. Myocardial infarction & 11 \\
\hline 2. Congestive heart failure & 3 \\
\hline 3. Peripheral vascular disease & 10 \\
\hline 4. Cerebrovascular disease & 9 \\
\hline 5. Dementia & 1 \\
\hline 6. COPD & 15 \\
\hline 7. Connective tissue disease & 12 \\
\hline 8. Peptic ulcer disease & 14 \\
\hline 9. Diabetes mellitus (1 point uncomplicated, 2 points if end-organ damage) & 64 \\
\hline 10. Moderate to severe chronic kidney disease (2 points) & 198 \\
\hline 11. Hemiplegia (2 points) & 0 \\
\hline 12. Leukemia (2 points) & 0 \\
\hline 13. Malignant lymphoma (2 points) & 0 \\
\hline 14. Solid tumour (2 points, 6 points if metastatic) & 14 \\
\hline 15. Liver disease (1 point mild, 3 points if moderate to severe) & 18 \\
\hline 16. AIDS (6 points) & 0 \\
\hline
\end{tabular}




\begin{tabular}{|l|c|c|}
\hline Table 4. Description and rates of all complications in the 90-day postoperative period \\
\hline 90-day complications breakdowns & $\mathbf{N}$ & $\mathbf{\%}$ \\
\hline CARDIAC COMPLICATIONS & $\mathbf{2 2}$ & $\mathbf{1 1 . 1}$ \\
\hline Hypertensive urgency & 1 & 0.5 \\
\hline Pericarditis & 1 & 0.5 \\
\hline Cardiac arrest & 2 & 1.0 \\
\hline NSTEMI & 3 & 1.5 \\
\hline Hypotension & 2 & 1.0 \\
\hline Cardiogenic shock & 2 & 1.0 \\
\hline Supraventricular Tachycardia (SVT) & 1 & 0.5 \\
\hline Atrial Fibrillation & 2 & 1.0 \\
\hline Hypertension (new onset requiring medical therapy) & 8 & 4.0 \\
\hline RESPIRATORY COMPLICATIONS & $\mathbf{2 0}$ & $\mathbf{1 0 . 1}$ \\
\hline Respiratory failure & 4 & 2.0 \\
\hline Pleural effusion & 1 & 0.5 \\
\hline Viral URTI/LRTI & 2 & 1.0 \\
\hline Atelectasis & 1 & 0.5 \\
\hline Pulmonary embolism (PE) & 1 & 0.5 \\
\hline Pulmonary edema & 6 & 3.0 \\
\hline Pneumonia & 3 & 2.5 \\
\hline GI COMPLICATIONS & 3 & 1.5 \\
\hline C. difficile infection & 32 & 16.2 \\
\hline Chronic abdominal pain & 1 & 0.5 \\
\hline Diarrhea, resolving after immunosuppressant adjustment & 3 & 0.5 \\
\hline Dysphagia & 3 \\
\hline Gastroenteritis & 1.5 \\
\hline GI bleed & 2 \\
\hline
\end{tabular}




\begin{tabular}{|l|c|c|}
\hline Ileus & 8 & 4.0 \\
\hline Nausea/Vomiting & 5 & 2.5 \\
\hline Pancreatic leak & 1 & 0.5 \\
\hline Small Bowel Obstruction (SBO) & 3 & 1.5 \\
\hline Constipation, chronic & 1 & 0.5 \\
\hline Pill esophagitis & 1 & 0.5 \\
\hline NEUROLOGIC COMPLICATIONS & $\mathbf{2}$ & $\mathbf{1 . 0}$ \\
\hline Zoster & 1 & 0.5 \\
\hline Subarachnoid Hemorrhage & 1 & 0.5 \\
\hline HEMATOLOGIC COMPLICATIONS & $\mathbf{4 3}$ & $\mathbf{2 1 . 7}$ \\
\hline Transfusion of Packed Red Blood Cells & 38 & 19.2 \\
\hline Deep Vein Thrombosis (DVT) & 4 & 2.0 \\
\hline Hemolytic drug reaction & 1 & 0.5 \\
\hline METABOLIC COMPLICATIONS & $\mathbf{1 5}$ & $\mathbf{7 . 6}$ \\
\hline Hyperkalemia (requiring HD) & 2 & 1.0 \\
\hline Post-transplant diabetes & 7 & 3.5 \\
\hline Hypercalcemia (severe) & 1 & 0.5 \\
\hline Hypomagnesemia (severe) & 1 & 0.5 \\
\hline Hypoglycemic emergency & 1 & 0.5 \\
\hline Tertiary hyperparathyroidism & $\mathbf{2}$ & 0.5 \\
\hline Drug induced transaminitis & 7 & 0.5 \\
\hline Transient hyperglycemia & $\mathbf{1 7 . 7}$ \\
\hline INFECTIOUS COMPLICATIONS & 3.5 \\
\hline Sepsis & 11 & 5.6 \\
\hline Urinary Tract Infection & 2.5 \\
\hline Graft bed infection (abscess/infected lymphocele or hematoma) & 1 & 0.5 \\
\hline Wound infection & 1 & \\
\hline
\end{tabular}




\begin{tabular}{|c|c|c|}
\hline Immunosuppressant related infection (BK viruria, CMV viremia) & 5 & 2.5 \\
\hline Cervical lymphadenitits & 1 & 0.5 \\
\hline Esophageal candidiasis & 1 & 0.5 \\
\hline Oral thrush & 1 & 0.5 \\
\hline GRAFT COMPLICATIONS & 19 & 9.6 \\
\hline Calcineurin inhibitor toxicity/complication & 2 & 1.0 \\
\hline Ureteric obstruction (clot, stricture) & 4 & 2.0 \\
\hline Perigraft collections (lymphocele; hematoma) - symptomatic & 8 & 4.0 \\
\hline Ureteric leak & 1 & 0.5 \\
\hline Gross hematuria & 1 & 0.5 \\
\hline Wound dehiscence & 1 & 0.5 \\
\hline Glomerulonephritis recurrence & 1 & 0.5 \\
\hline Wound seroma & 1 & 0.5 \\
\hline OTHER COMPLICATIONS & 15 & 7.6 \\
\hline Delayed mobilization requiring rehab team and extending stay & 1 & 0.5 \\
\hline Poor pain control requiring Acute Pain Service & 1 & 0.5 \\
\hline Panic attack & 1 & 0.5 \\
\hline Fall & 1 & 0.5 \\
\hline Gout flair & 3 & 1.5 \\
\hline Steroid induced psychosis & 1 & 0.5 \\
\hline Urge incontinence (new) & 2 & 1.0 \\
\hline Reactive arthritis & 1 & 0.5 \\
\hline Sacral ulcer & 1 & 0.5 \\
\hline Urethral injury (Foley malposition) & 1 & 0.5 \\
\hline Urinary retention & 2 & 1.0 \\
\hline
\end{tabular}

